\title{
Generalized weighted composition operators from $\alpha$-Bloch spaces into weighted-type spaces
}

Songxiao $\mathrm{Li}^{1}$ and Stevo Stević $2,3^{*}$

"Correspondence: sstevic@ptt.rs ${ }^{2}$ Mathematical Institute of the Serbian Academy of Sciences, Knez Mihailova 36/III, Beograd, 11000, Serbia

${ }^{3}$ Operator Theory and Applications Research Group, Department of Mathematics, King Abdulaziz University, P.O. Box 80203, Jeddah, 21589, Saudi Arabia

Full list of author information is available at the end of the article

\begin{abstract}
Some criteria for the boundedness, as well as for the compactness, of the generalized weighted composition operator $D_{\varphi, u}^{n}$ from $\alpha$-Bloch spaces into weighted-type spaces are given. Estimates for the norm and the essential norm of the operator are also given. Our results extend and complement some results in the literature.
\end{abstract}

MSC: 47B33; $30 \mathrm{H} 30$

Keywords: generalized weighted composition operators; weighted-type space; $\alpha$-Bloch space; boundedness; compactness; essential norm

\section{Introduction}

Let $\mathbb{D}$ be the unit disk of the complex plane $\mathbb{C}, H(\mathbb{D})$ the class of functions analytic on $\mathbb{D}$, and $H^{\infty}=H^{\infty}(\mathbb{D})$ the space of bounded analytic functions on $\mathbb{D}$. For $0<\alpha<\infty$, an $f \in H(\mathbb{D})$ is said to belong to the $\alpha$-Bloch space $\mathcal{B}^{\alpha}=\mathcal{B}^{\alpha}(\mathbb{D})$ if

$$
b_{\alpha}(f)=\sup _{z \in \mathbb{D}}\left(1-|z|^{2}\right)^{\alpha}\left|f^{\prime}(z)\right|<\infty .
$$

It is easy to check that $\mathcal{B}^{\alpha}$ becomes a Banach space with the norm $\|f\|_{\mathcal{B}^{\alpha}}=|f(0)|+b_{\alpha}(f)$. The little $\alpha$-Bloch space $\mathcal{B}_{0}^{\alpha}=\mathcal{B}_{0}^{\alpha}(\mathbb{D})$, is a subspace of $\mathcal{B}^{\alpha}$ consisting of all $f \in H(\mathbb{D})$ such that

$$
\lim _{|z| \rightarrow 1^{-}}\left(1-|z|^{2}\right)^{\alpha}\left|f^{\prime}(z)\right|=0
$$

When $\alpha=1, \mathcal{B}^{1}=\mathcal{B}$ is the well-known Bloch space, while $\mathcal{B}_{0}^{1}=\mathcal{B}_{0}$ is the well-known little Bloch space. For some results on the $\alpha$-Bloch spaces and the little $\alpha$-Bloch spaces, see, for example, [1].

A positive continuous function on $\mathbb{D}$ is called a weight. Let $\mu(z)$ be a weight. The weighted-type space on $\mathbb{D}[2,3]$, denoted by $H_{\mu}^{\infty}=H_{\mu}^{\infty}(\mathbb{D})$, consists of all $f \in H(\mathbb{D})$ such that

$$
\|f\|_{H_{\mu}^{\infty}}=\sup _{z \in \mathbb{D}} \mu(z)|f(z)|<\infty .
$$

\section{Springer}

(c) $2015 \mathrm{Li}$ and Stević. This article is distributed under the terms of the Creative Commons Attribution 4.0 International License (http://creativecommons.org/licenses/by/4.0/), which permits unrestricted use, distribution, and reproduction in any medium, provided you give appropriate credit to the original author(s) and the source, provide a link to the Creative Commons license, and indicate if changes were made. 
It is obvious that $H_{0}^{\infty}=H^{\infty}$, while for $\mu(z)=\left(1-|z|^{2}\right)^{\beta}, \beta>0$, is obtained the growth space $H_{\beta}^{\infty}[4]$.

Let $u \in H(\mathbb{D})$ and $\varphi$ be an analytic self-map of $\mathbb{D}$. The weighted composition operator $u C_{\varphi}$, induced by $\varphi$ and $u$, is defined by

$$
\left(u C_{\varphi} f\right)(z)=u(z) \cdot f(\varphi(z)), \quad f \in H(\mathbb{D}), z \in \mathbb{D} .
$$

When $u(z) \equiv 1$, then the weighted composition operator is reduced to the composition operator, usually denoted by $C_{\varphi}$, while for $\varphi(z) \equiv z$, it is reduced to the multiplication operator, usually denoted by $M_{u}$.

A natural generalization of the weighted composition operator is the generalized weighted composition operator [5] or the weighted differentiation composition operator [6] $D_{\varphi, u}^{n}$, which is defined as

$$
\left(D_{\varphi, u}^{n} f\right)(z)=u(z) \cdot f^{(n)}(\varphi(z)), \quad f \in H(\mathbb{D}), z \in \mathbb{D},
$$

where $n \in \mathbb{N}_{0}, u \in H(\mathbb{D})$, and $\varphi$ is an analytic self-map of $\mathbb{D}$. Clearly, when $n=0$ and $u(z)=1, D_{\varphi, u}^{n}$ is the composition operator $C_{\varphi}$, if $n=0$, then $D_{\varphi, u}^{n}$ is the weighted composition operator $u C_{\varphi}$. If $n=1$ and $u(z)=\varphi^{\prime}(z)$, then $D_{\varphi, u}^{n}=D C_{\varphi}$, which was studied, for example, in [3, 7-15], while for $u(z)=1, D_{\varphi, u}^{n}=C_{\varphi} D^{n}$, which was studied in $[3,13,15,16]$. For some other results on the generalized weighted composition operator on various spaces of holomorphic functions, see, for example, [17-22]. A fundamental problem concerning concrete operators is to relate function theoretic properties of their symbols to their operator theoretic properties (see, for example, [3, 5-29]).

It is well known that the composition operator is bounded on the Bloch space $\mathcal{B}$. See, for example, $[26,28,29]$ for the compactness and essential norm of the composition operator on $\mathcal{B}$. In [28], it was shown that $C_{\varphi}$ is compact on $\mathcal{B}$ if and only if

$$
\left\|C_{\varphi} p_{j}\right\|_{\mathcal{B}}=\left\|\varphi^{j}\right\|_{\mathcal{B}} \rightarrow 0 \quad \text { as } j \rightarrow \infty
$$

where $p_{j}(z)=z^{j}, j \in \mathbb{N}_{0}$.

Motivated by this result, in [22], the author proved that $D_{\varphi, u}^{n}: \mathcal{B} \rightarrow H_{\beta}^{\infty}$ is compact if and only if it is bounded and

$$
\lim _{j \rightarrow \infty}\left\|D_{\varphi, u}^{n}\left(p_{j}\right)\right\|_{H_{\beta}^{\infty}}=0
$$

Following the line of the above mentioned investigations, in this work, we consider the operators $D_{\varphi, u}^{n}: \mathcal{B}^{\alpha}\left(\right.$ or $\left.\mathcal{B}_{0}^{\alpha}\right) \rightarrow H_{\mu}^{\infty}$, and show that $D_{\varphi, u}^{n}: \mathcal{B}^{\alpha}\left(\right.$ or $\left.\mathcal{B}_{0}^{\alpha}\right) \rightarrow H_{\mu}^{\infty}$ is bounded (respectively, compact) if and only if the sequence $\left(j^{\alpha-1}\left\|D_{\varphi, u}^{n}\left(p_{j}\right)\right\|_{H_{\mu}^{\infty}}^{\infty}\right)_{j=n}^{\infty}$ is bounded (respectively, convergent to 0 as $j \rightarrow \infty$ ). Moreover, we give some estimates for the norm, as well as for the essential norm of the operator $D_{\varphi, u}^{n}: \mathcal{B}^{\alpha}\left(\right.$ or $\left.\mathcal{B}_{0}^{\alpha}\right) \rightarrow H_{\mu}^{\infty}$. Recall that the essential norm of the operator $T: X \rightarrow Y$ is its distance to the set of compact operators $K$ mapping $X$ to $Y$, that is,

$$
\|T\|_{e, X \rightarrow Y}=\inf \left\{\|T-K\|_{X \rightarrow Y}: K \text { is compact }\right\},
$$


where $X$ and $Y$ are Banach spaces and $\|\cdot\|_{X \rightarrow Y}$ is the operator norm. Consequently, $\|T\|_{e, X \rightarrow Y}=0$ if and only if $T$ is compact.

Throughout the paper, we denote by $C$ a positive constant which may differ from one occurrence to the next. We write $P \preceq Q$ if there exists a positive constant $C$ independent of the quantities $P$ and $Q$ such that $P \leq C Q$. The symbol $P \approx Q$ means that $P \preceq Q \preceq P$.

\section{Boundedness of $D_{\varphi, u}^{n}: \mathcal{B}^{\alpha}\left(\right.$ or $\left.\mathcal{B}_{0}^{\alpha}\right) \rightarrow H_{\mu}^{\infty}$}

For $w \in \mathbb{D}$, set

$$
f_{w}(z)=\frac{1-|w|^{2}}{(1-\bar{w} z)^{\alpha}}, \quad z \in \mathbb{D}
$$

Note that

$$
f_{w}^{(n)}(z)=\frac{\left(1-|w|^{2}\right) \bar{w}^{n}}{(1-\bar{w} z)^{\alpha+n}} \prod_{j=0}^{n-1}(\alpha+j), \quad z \in \mathbb{D}, n \in \mathbb{N} .
$$

In this section, we will use this family of functions, as well as the sequence of functions $\left(j^{\alpha-1} p_{j}\right)_{j \in \mathbb{N}}$ to characterize the boundedness and compactness of $D_{\varphi, u}^{n}: \mathcal{B}^{\alpha}\left(\right.$ or $\left.\mathcal{B}_{0}^{\alpha}\right) \rightarrow H_{\mu}^{\infty}$.

Theorem 2.1 Let $n$ be a positive integer, $\alpha>0, \mu$ a weight, $u \in H(\mathbb{D})$, and $\varphi$ be an analytic self-map of $\mathbb{D}$. Then the following statements are equivalent.

(a) The operator $D_{\varphi, u}^{n}: \mathcal{B}^{\alpha} \rightarrow H_{\mu}^{\infty}$ is bounded.

(b) The operator $D_{\varphi, u}^{n}: \mathcal{B}_{0}^{\alpha} \rightarrow H_{\mu}^{\infty}$ is bounded.

(c) $M_{1}:=\sup _{j \geq n} j^{\alpha-1}\left\|D_{\varphi, u}^{n}\left(p_{j}\right)\right\|_{H_{\mu}^{\infty}}<\infty$.

(d) $M_{2}:=\sup _{w \in \mathbb{D}}\left\|D_{\varphi, u}^{n} f_{\varphi(w)}\right\|_{H_{\mu}^{\infty}}<\infty$ and $u \in H_{\mu}^{\infty}$.

(e) $M_{3}:=\sup _{z \in \mathbb{D}} \frac{\mu(z)|u(z)|}{\left(1-|\varphi(z)|^{2}\right)^{n+\alpha-1}}<\infty$ and $u \in H_{\mu}^{\infty}$.

Moreover, if the operator $D_{\varphi, u}^{n}: \mathcal{B}^{\alpha} \rightarrow H_{\mu}^{\infty}$ is bounded, then the following asymptotic relations hold:

$$
\left\|D_{\varphi, u}^{n}\right\|_{\mathcal{B}^{\alpha} \rightarrow H_{\mu}^{\infty}} \approx\left\|D_{\varphi, u}^{n}\right\|_{\mathcal{B}_{0}^{\alpha} \rightarrow H_{\mu}^{\infty}} \approx M_{1} \approx \max \left\{M_{2},\|u\|_{H_{\mu}^{\infty}}\right\} \approx M_{3} .
$$

Proof (a) $\Rightarrow$ (b) Since $\mathcal{B}_{0}^{\alpha} \subset \mathcal{B}^{\alpha}$, this implication, as well as the inequality

$$
\left\|D_{\varphi, u}^{n}\right\|_{\mathcal{B}_{0}^{\alpha} \rightarrow H_{\mu}^{\infty}} \leq\left\|D_{\varphi, u}^{n}\right\|_{\mathcal{B}^{\alpha} \rightarrow H_{\mu}^{\infty}}
$$

is obvious.

(b) $\Rightarrow$ (c) It is easy to see that the sequence $\left(j^{\alpha-1} p_{j}\right)_{j \in \mathbb{N}}$ is bounded in $\mathcal{B}_{0}^{\alpha}$ and

$$
\left\|p_{j}\right\|_{\mathcal{B}^{\alpha}}=j\left(\frac{2 \alpha}{j-1+2 \alpha}\right)^{\alpha}\left(\frac{j-1}{j-1+2 \alpha}\right)^{\frac{j-1}{2}}, \quad \text { for } j \in \mathbb{N},
$$

which implies that $\left\|j^{\alpha-1} p_{j}\right\|_{\mathcal{B}^{\alpha}} \approx 1$. Notice that $\left(D_{\varphi, u}^{n} p_{n}\right)(z)=u(z) n !, z \in \mathbb{D}$, while for $j<n$, $D_{\varphi, u}^{n}\left(p_{j}\right)=0$. Therefore, by the boundedness of $D_{\varphi, u}^{n}: \mathcal{B}_{0}^{\alpha} \rightarrow H_{\mu}^{\infty}$, we get

$$
j^{\alpha-1}\left\|D_{\varphi, u}^{n}\left(p_{j}\right)\right\|_{H_{\mu}^{\infty}}=\left\|D_{\varphi, u}^{n}\left(j^{\alpha-1} p_{j}\right)\right\|_{H_{\mu}^{\infty}} \leq C\left\|D_{\varphi, u}^{n}\right\|_{\mathcal{B}_{0}^{\alpha} \rightarrow H_{\mu}^{\infty}}<\infty
$$


for every $j \in \mathbb{N}$, proving (c), as well as the asymptotic relation

$$
M_{1} \preceq\left\|D_{\varphi, u}^{n}\right\|_{\mathcal{B}_{0}^{\alpha} \rightarrow H_{\mu}^{\infty}}
$$

(c) $\Rightarrow$ (a) If $\|\varphi\|_{\infty}=\sup _{z \in \mathbb{D}}|\varphi(z)|<1$, then by Proposition 8 in [1], we have

$$
\left\|D_{\varphi, u}^{n} f\right\|_{H_{\mu}^{\infty}} \preceq \frac{\|u\|_{H_{\mu}^{\infty}}\|f\|_{\mathcal{B}^{\alpha}}}{\left(1-\|\varphi\|_{\infty}^{2}\right)^{n+\alpha-1}},
$$

from which the boundedness of $D_{\varphi, u}^{n}: \mathcal{B}^{\alpha} \rightarrow H_{\mu}^{\infty}$ follows in this case.

Now assume that $\|\varphi\|_{\infty}=1$. Let $\mathbb{D}_{j}=\left\{z \in \mathbb{D}: r_{j} \leq|\varphi(z)|<r_{j+1}\right\}$ where $r_{j}=(j-n) /(j+\alpha-1)$ for $j \geq n$. Then from Lemma 1 in [16], which also holds for $m=0$, i.e., $n=1$ in our case, we have that there is a $\delta>0$ such that

$$
\min _{z \in \mathbb{D}} j^{\alpha-1} j(j-1) \cdots(j-n+1)|\varphi(z)|^{j-n}(1-|\varphi(z)|)^{\alpha+n-1} \geq \delta,
$$

for every $j \geq k+1$, where $k$ is the smallest natural number such that $\mathbb{D}_{k} \neq \emptyset$.

Fix $N \geq k+1$. Then, clearly $N \geq n+1$ and we have

$$
\left\|D_{\varphi, U}^{n} f\right\|_{H_{\mu}^{\infty}} \leq \sup _{|\varphi(z)|<\frac{N-n}{N+\alpha-1}} \mu(z)|u(z)|\left|f^{(n)}(\varphi(z))\right|+\sup _{|\varphi(z)| \geq \frac{N-n}{N+\alpha-1}} \mu(z)|u(z)|\left|f^{(n)}(\varphi(z))\right| .
$$

The finiteness of $M_{1}$ implies $u \in H_{\mu}^{\infty}$. Hence, as in the first case, we have

$$
\sup _{|\varphi(z)|<\frac{N-n}{N+\alpha-1}} \mu(z)|u(z)|\left|f^{(n)}(\varphi(z))\right| \preceq\|u\|_{H_{\mu}^{\infty}}\|f\|_{\mathcal{B}^{\alpha}} .
$$

On the other hand, since $\mathbb{D} \backslash\left\{|\varphi(z)|<\frac{N-n}{N+\alpha-1}\right\}=\bigcup_{j \geq N} \mathbb{D}_{j}$, we get

$$
\begin{aligned}
& \sup _{|\varphi(z)| \geq N-n)} \mu(z)|u(z)|\left|f^{(n)}(\varphi(z))\right| \\
& =\sup _{j+\alpha-1} \sup _{z \in \mathbb{D}_{j}} \mu(z)|u(z)|\left|f^{(n)}(\varphi(z))\right| \\
& =\sup _{j \geq N} \sup _{z \in \mathbb{D}_{j}} \mu(z)|u(z)| \frac{j^{\alpha-1} j(j-1) \cdots(j-n+1)|\varphi(z)|^{j-n}\left|f^{(n)}(\varphi(z))\right|(1-|\varphi(z)|)^{\alpha+n-1}}{j^{\alpha-1} j(j-1) \cdots(j-n+1)(1-|\varphi(z)|)^{\alpha+n-1}|\varphi(z)|^{j-n}} \\
& \leq \frac{\|f\|_{\mathcal{B}^{\alpha}}}{\delta} \sup _{j \geq N} j^{\alpha-1}\left\|D_{\varphi, u}^{n}\left(p_{j}\right)\right\|_{H_{\mu}^{\infty}} \leq \frac{M_{1}}{\delta}\|f\|_{\mathcal{B}^{\alpha}<\infty .}
\end{aligned}
$$

From (5), (6) and (7), the boundedness of $D_{\varphi, u}^{n}: \mathcal{B}^{\alpha} \rightarrow H_{\mu}^{\infty}$ follows.

(c) $\Rightarrow$ (d) First note that (c) implies that $u \in H_{\mu}^{\infty}$. Further, since

$$
\sup _{z \in \mathbb{D}}\left(1-|z|^{2}\right)^{\alpha}\left|f_{w}^{\prime}(z)\right|=\sup _{z \in \mathbb{D}}\left(1-|z|^{2}\right)^{\alpha} \frac{|\alpha \bar{w}|\left(1-|w|^{2}\right)}{|1-\bar{w} z|^{\alpha+1}} \leq|\alpha| 2^{\alpha+1}, \quad w \in \mathbb{D},
$$

the family of functions $\left(f_{w}\right)_{w \in \mathbb{D}}$ is uniformly bounded in $\mathcal{B}^{\alpha}$. Furthermore

$$
f_{w}(z)=\left(1-|w|^{2}\right) \sum_{j=0}^{\infty} \frac{\Gamma(j+\alpha)}{j ! \Gamma(\alpha)} \bar{w}^{j} z^{j}, \quad z \in \mathbb{D} .
$$


By Stirling's formula, we have $\frac{\Gamma(j+\alpha)}{j ! \Gamma(\alpha)} \approx j^{\alpha-1}$ as $j \rightarrow \infty$. Using this fact, the linearity and continuity of the operator, we get

$$
\left\|D_{\varphi, u}^{n} f_{w}\right\|_{H_{\mu}^{\infty}} \leq C\left(1-|w|^{2}\right) \sum_{j=n}^{\infty}|w|^{j} j^{\alpha-1}\left\|D_{\varphi, u}^{n}\left(p_{j}\right)\right\|_{H_{\mu}^{\infty}} \preceq M_{1}<\infty, \quad w \in \mathbb{D} .
$$

Consequently, $\sup _{w \in \mathbb{D}}\left\|D_{\varphi, u}^{n} f_{\varphi(w)}\right\|_{H_{\mu}^{\infty}} \preceq M_{1}$, and along with the inequality $n^{\alpha-1} n !\|u\|_{H_{\mu}^{\infty}} \leq$ $M_{1}$, obtained by considering $\left\|D_{\varphi, u}^{n}\left(n^{\alpha-1} p_{n}\right)\right\|_{H_{\mu}^{\infty}}$, we also have

$$
\max \left\{M_{2},\|u\|_{H_{\mu}^{\infty}}\right\} \preceq M_{1} .
$$

(d) $\Rightarrow$ (e) For $\lambda \in \mathbb{D}$, it follows from (d) and (1) that

$$
M_{2} \geq\left\|D_{\varphi, u}^{n} f_{\varphi(\lambda)}\right\|_{H_{\mu}^{\infty}} \geq \frac{\mu(\lambda)|u(\lambda) \| \varphi(\lambda)|^{n} \prod_{j=0}^{n-1}(\alpha+j)}{\left(1-|\varphi(\lambda)|^{2}\right)^{n+\alpha-1}} .
$$

For any fixed $r \in(0,1)$, from (9), we have

$$
\sup _{|\varphi(\lambda)|>r} \frac{\mu(\lambda)|u(\lambda)|}{\left(1-|\varphi(\lambda)|^{2}\right)^{n+\alpha-1}} \leq \sup _{|\varphi(\lambda)|>r} \frac{|\varphi(\lambda)|^{n}}{r^{n}} \frac{\mu(\lambda)|u(\lambda)|}{\left(1-|\varphi(\lambda)|^{2}\right)^{n+\alpha-1}} \preceq \frac{M_{2}}{r^{n}} .
$$

On the other hand, from $u \in H_{\mu}^{\infty}$, we have

$$
\begin{aligned}
\sup _{|\varphi(\lambda)| \leq r} \frac{\mu(\lambda)|u(\lambda)|}{\left(1-|\varphi(\lambda)|^{2}\right)^{n+\alpha-1}} & \leq \frac{\sup _{|\varphi(\lambda)| \leq r} \mu(\lambda)|u(\lambda)|}{\left(1-r^{2}\right)^{n+\alpha-1}} \\
& \leq \frac{\|u\|_{H_{\mu}^{\infty}}}{\left(1-r^{2}\right)^{n+\alpha-1}}<\infty .
\end{aligned}
$$

Therefore, (10) and (11) yield the inequality of (e), as well as the asymptotic relation

$$
M_{3} \preceq \max \left\{M_{2},\|u\|_{H_{\mu}^{\infty}}\right\} .
$$

(e) $\Rightarrow$ (a) By Proposition 8 in [1], if $f \in \mathcal{B}^{\alpha}$ and $k \in \mathbb{N}$, we see that

$$
\sup _{z \in \mathbb{D}}\left(1-|z|^{2}\right)^{k+\alpha-1}\left|f^{(k)}(z)\right| \leq C\|f\|_{\mathcal{B}^{\alpha}},
$$

for some constant $C$ independent of $f$. Therefore, for $z \in \mathbb{D}$, we have

$$
\begin{aligned}
\mu(z)\left|\left(D_{\varphi, u}^{n} f\right)(z)\right| & =\mu(z)|u(z)|\left|f^{(n)}(\varphi(z))\right| \\
& \leq C \frac{\mu(z)|u(z)|}{\left(1-|\varphi(z)|^{2}\right)^{n+\alpha-1}}\|f\|_{\mathcal{B}^{\alpha}},
\end{aligned}
$$

where $C$ is independent of $f$. Taking the supremum in (13) over $\mathbb{D}$ and then using the first condition in (e) we see that $D_{\varphi, u}^{n}: \mathcal{B}^{\alpha} \rightarrow H_{\mu}^{\infty}$ is bounded, and

$$
\left\|D_{\varphi, u}^{n}\right\|_{\mathcal{B}^{\alpha} \rightarrow H_{\mu}^{\infty}} \preceq M_{3} .
$$

If the operator $D_{\varphi, u}^{n}: \mathcal{B}^{\alpha} \rightarrow H_{\mu}^{\infty}$ is bounded, then from (3), (4), (8), (12), and (14), we obtain (2), completing the proof. 


\section{Compactness and essential norm of $D_{\varphi, u}^{n}: \mathcal{B}^{\alpha}\left(\right.$ or $\left.\mathcal{B}_{0}^{\alpha}\right) \rightarrow H_{\mu}^{\infty}$}

In this section we will give an estimate for the essential norm of the operator $D_{\varphi, u}^{n}: \mathcal{B}^{\alpha} \rightarrow$ $H_{\mu}^{\infty}$, as well as of $D_{\varphi, u}^{n}: \mathcal{B}_{0}^{\alpha} \rightarrow H_{\mu}^{\infty}$. For this purpose, we state several lemmas, which will be used in the proof of the main result.

Lemma 3.1 [16] Let $\alpha>0, m \geq n+1$, where $n \in \mathbb{N}$. Define the function $H_{m, \alpha}:[0,1] \rightarrow$ $[0, \infty)$ by

$$
H_{m, \alpha}(x)=\frac{m !}{(m-n-1) !} x^{m-n-1}(1-x)^{n+\alpha} .
$$

Then the following statements hold:

(i)

$$
\max _{0 \leq x \leq 1} H_{m, \alpha}(x)=H_{m, \alpha}\left(r_{m}\right)= \begin{cases}(n+1) !, & m=n+1, \\ \frac{m !}{(m-n-1) !}\left(\frac{m-n-1}{m+\alpha-1}\right)^{m-n-1}\left(\frac{n+\alpha}{m+\alpha-1}\right)^{\alpha+n}, & m>n+1,\end{cases}
$$

where

$$
r_{m}= \begin{cases}0, & m=n+1 \\ \frac{m-n-1}{m+\alpha-1}, & m>n+1\end{cases}
$$

(ii) For $m>n+1, H_{m, \alpha}$ is decreasing on $\left[r_{m}, r_{m+1}\right]$, and so

$$
\min _{r_{m} \leq x \leq r_{m+1}} H_{m, \alpha}(x)=H_{m, \alpha}\left(r_{m+1}\right)=\frac{m !}{(m-n-1) !}\left(\frac{m-n}{m+\alpha}\right)^{m-n-1}\left(\frac{n+\alpha}{m+\alpha}\right)^{\alpha+n} .
$$

Consequently,

$$
\lim _{m \rightarrow \infty} m^{\alpha-1} \min _{r_{m} \leq x \leq r_{m+1}} H_{m, \alpha}(x)=\frac{(n+\alpha)^{n+\alpha}}{e^{n+\alpha}} .
$$

Denote by $K_{r} f(z)=f(r z)$ for $r \in(0,1)$ and $z \in \mathbb{D}$. Then $K_{r}$ is a compact operator on $\mathcal{B}^{\alpha}$ for every $\alpha>0$, and $\left\|K_{r}\right\| \leq 1$ (see, e.g., Proposition 1.3 in [24] and [27]). Let $I$ denote the identity operator. The following three lemmas can be found in [25] (see also [16]).

Lemma 3.2 Let $0<\alpha<1$. Then there is a sequence $\left(r_{k}\right)_{k \in \mathbb{N}}$, with $0<r_{k}<1$ tending to 1 , such that the sequence of compact operators $L_{j}=\frac{1}{j} \sum_{k=1}^{j} K_{r_{k}}, j \in \mathbb{N}$, on $\mathcal{B}_{0}^{\alpha}$ satisfies the following.

(i) For any $t \in(0,1), \lim _{j \rightarrow \infty} \sup _{|| f \|_{\mathcal{B}^{\alpha} \leq 1}} \sup _{|z| \leq t}\left|\left(\left(I-L_{j}\right) f\right)^{\prime}(z)\right|=0$.

(ii) $\lim _{j \rightarrow \infty} \sup _{\|f\|_{\mathcal{B}^{\alpha} \leq 1}} \sup _{z \in \mathbb{D}}\left|\left(I-L_{j}\right) f(z)\right|=0$.

(iii) $\lim \sup _{j \rightarrow \infty}\left\|I-L_{j}\right\| \leq 1$.

Furthermore, these statements hold as well for the sequence of biadjoints $L_{j}^{* *}$ on $\mathcal{B}^{\alpha}$.

Lemma 3.3 Let $\alpha=1$. Then there is a sequence $\left(r_{k}\right)_{k \in \mathbb{N}}$, with $0<r_{k}<1$ tending to 1 , such that the sequence of compact operators $L_{j}=\frac{1}{j} \sum_{k=1}^{j} K_{r_{k}}, j \in \mathbb{N}$, on $\mathcal{B}_{0}$ satisfies the following.

(i) For any $t \in[0,1), \lim _{j \rightarrow \infty} \sup _{\|f\|_{\mathcal{B} \leq 1}} \sup _{|z| \leq t}\left|\left(\left(I-L_{j}\right) f\right)^{\prime}(z)\right|=0$.

(iia) $\lim _{j \rightarrow \infty} \sup _{\|f\|_{\mathcal{B}} \leq 1} \sup _{|z|>s}\left|\left(I-L_{j}\right) f(z)\right|\left(\log \frac{1}{1-|z|^{2}}\right)^{-1} \leq 1$, for s sufficiently close to 1 .

(iib) $\lim _{j \rightarrow \infty} \sup _{|| f \|_{\mathcal{B} \leq 1}} \sup _{|z| \leq s}\left|\left(I-L_{j}\right) f(z)\right|=0$ for the above $s$.

(iii) $\lim \sup _{j \rightarrow \infty}\left\|I-L_{j}\right\| \leq 1$.

Furthermore, these statements hold as well for the sequence of biadjoints $L_{j}^{* *}$ on $\mathcal{B}$. 
Lemma 3.4 Let $\alpha>1$. Then there is a sequence $\left(r_{k}\right)_{k \in \mathbb{N}}$, with $0<r_{k}<1$ tending to 1 , such that the sequence of compact operators $L_{j}=\frac{1}{j} \sum_{k=1}^{j} K_{r_{k}}, j \in \mathbb{N}$, on $\mathcal{B}_{0}^{\alpha}$ satisfies the following.

(i) For any $t \in[0,1), \lim _{j \rightarrow \infty} \sup _{\|f\|_{\mathcal{B}^{\alpha} \leq 1}} \sup _{|z| \leq t}\left|\left(\left(I-L_{j}\right) f\right)^{\prime}(z)\right|=0$.

(ii) For any $t \in[0,1), \lim _{j \rightarrow \infty} \sup _{\|f\|_{\mathcal{B}^{\alpha} \leq 1}} \sup _{|z| \leq t}\left|\left(I-L_{j}\right) f(z)\right|=0$.

(iii) $\lim \sup _{j \rightarrow \infty}\left\|I-L_{j}\right\| \leq 1$.

Furthermore, these statements hold as well for the sequence of biadjoints $L_{j}^{* *}$ on $\mathcal{B}^{\alpha}$.

To study the compactness, we also need the following lemma, which can be proved in a standard way (see, for example, Proposition 3.11 in [23]).

Lemma 3.5 Let $n$ be a nonnegative integer, $\alpha>0, \mu$ a weight, $u \in H(\mathbb{D})$ and $\varphi$ be an analytic self-map of $\mathbb{D}$. Then $D_{\varphi, u}^{n}: \mathcal{B}^{\alpha}\left(\right.$ or $\left.\mathcal{B}_{0}^{\alpha}\right) \rightarrow H_{\mu}^{\infty}$ is compact if and only if $D_{\varphi, u}^{n}$ : $\mathcal{B}^{\alpha}\left(\right.$ or $\left.\mathcal{B}_{0}^{\alpha}\right) \rightarrow H_{\mu}^{\infty}$ is bounded and for any bounded sequence $\left(f_{k}\right)_{k \in \mathbb{N}}$ in $\mathcal{B}^{\alpha}$, which converges to zero uniformly on compact subsets of $\mathbb{D}$,

$$
\lim _{k \rightarrow \infty}\left\|D_{\varphi, u}^{n} f_{k}\right\|_{H_{\mu}^{\infty}}=0
$$

Now we are ready to state and prove the main results in this section.

Theorem 3.6 Let $n$ be a positive integer, $\alpha>0$, $\mu$ a weight, $u \in H(\mathbb{D})$, and $\varphi$ be an analytic self-map of $\mathbb{D}$. Suppose that $D_{\varphi, u}^{n}: \mathcal{B}^{\alpha} \rightarrow H_{\mu}^{\infty}$ is bounded. Then

$$
\left\|D_{\varphi, u}^{n}\right\|_{e, \mathcal{B}^{\alpha} \rightarrow H_{\mu}^{\infty}} \approx\left\|D_{\varphi, u}^{n}\right\|_{e, \mathcal{B}_{0}^{\alpha} \rightarrow H_{\mu}^{\infty}} \approx \limsup _{j \rightarrow \infty} j^{\alpha-1}\left\|D_{\varphi, u}^{n}\left(p_{j}\right)\right\|_{H_{\mu}^{\infty}}
$$

Proof First note that the inequality

$$
\left\|D_{\varphi, u}^{n}\right\|_{e, \mathcal{B}_{0}^{\alpha} \rightarrow H_{\mu}^{\infty}} \leq\left\|D_{\varphi, u}^{n}\right\|_{e, \mathcal{B}^{\alpha} \rightarrow H_{\mu}^{\infty}}
$$

obviously holds.

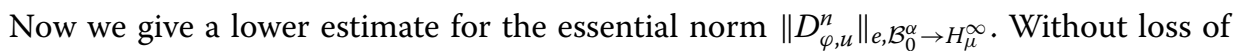
generality, we assume that $j \geq n$. Choose the sequence of functions $q_{j}=j^{\alpha-1} p_{j} \in \mathcal{B}_{0}^{\alpha}, j \in \mathbb{N}$. Then $\left\|q_{j}\right\|_{\mathcal{B}^{\alpha}} \approx 1$, and $\left(q_{j}\right)_{j \in \mathbb{N}}$ converges to zero weakly on $\mathcal{B}_{0}^{\alpha}$ as $j \rightarrow \infty$ (see, for example, Theorem 7.5 in [30]). Since by a well-known theorem, for any compact operator $\widehat{K}: X \rightarrow$ $Y$, where $X$ and $Y$ are Banach spaces, the weak convergence $x_{n} \stackrel{w}{\rightarrow} x_{0}$ implies the norm convergence $\widehat{K} x_{n} \rightarrow \widehat{K} x_{0}$ [31], we have

$$
\lim _{j \rightarrow \infty}\left\|K q_{j}\right\|_{H_{\mu}^{\infty}}=0
$$

for any given compact operator $K$ from $\mathcal{B}_{0}^{\alpha}$ to $H_{\mu}^{\infty}$.

Hence

$$
\left\|D_{\varphi, u}^{n}-K\right\|_{\mathcal{B}_{0}^{\alpha} \rightarrow H_{\mu}^{\infty}} \succeq\left\|\left(D_{\varphi, u}^{n}-K\right) q_{j}\right\|_{H_{\mu}^{\infty}} \geq\left\|D_{\varphi, u}^{n} q_{j}\right\|_{H_{\mu}^{\infty}}-\left\|K q_{j}\right\|_{H_{\mu}^{\infty}} .
$$

Letting $j \rightarrow \infty$ in the last relation and using (17), we obtain

$$
\left\|D_{\varphi, u}^{n}-K\right\|_{\mathcal{B}_{0}^{\alpha} \rightarrow H_{\mu}^{\infty}} \geq \limsup _{j \rightarrow \infty}\left\|D_{\varphi, u}^{n} q_{j}\right\|_{H_{\mu}^{\infty}}=\limsup _{j \rightarrow \infty} j^{\alpha-1}\left\|D_{\varphi, u}^{n}\left(p_{j}\right)\right\|_{H_{\mu}^{\infty}}
$$


and consequently

$$
\left\|D_{\varphi, u}^{n}\right\|_{e, \mathcal{B}_{0}^{\alpha} \rightarrow H_{\mu}^{\infty}}=\inf _{K}\left\|D_{\varphi, u}^{n}-K\right\|_{\mathcal{B}_{0}^{\alpha} \rightarrow H_{\mu}^{\infty}} \succeq \limsup _{j \rightarrow \infty} j^{\alpha-1}\left\|D_{\varphi, u}^{n}\left(p_{j}\right)\right\|_{H_{\mu}^{\infty}} .
$$

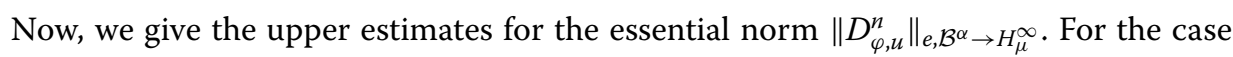
of $\sup _{z \in \mathbb{D}}|\varphi(z)|<1$, there is a number $\delta \in(0,1)$ such that $\sup _{z \in \mathbb{D}}|\varphi(z)|<\delta$. In this case, the operator $D_{\varphi, u}^{n}: \mathcal{B}^{\alpha} \rightarrow H_{\mu}^{\infty}$ is compact. Indeed, choose a bounded sequence $\left(f_{j}\right)_{j \in \mathbb{N}}$ in $\mathcal{B}^{\alpha}$ which converges to zero uniformly on compact subsets of $\mathbb{D}$. From Cauchy's integral formula, $\left(f_{j}^{(n)}\right)_{j \in \mathbb{N}}$ also converges to zero on compact subsets of $\mathbb{D}$ as $j \rightarrow \infty$. Hence

$$
\begin{aligned}
\lim _{j \rightarrow \infty}\left\|D_{\varphi, u}^{n} f_{j}\right\|_{H_{\mu}^{\infty}} & =\lim _{j \rightarrow \infty} \sup _{z \in \mathbb{D}} \mu(z)\left|u(z) f_{j}^{(n)}(\varphi(z))\right| \\
& \leq\|u\|_{H_{\mu}^{\infty}} \lim _{j \rightarrow \infty} \sup _{z \in \mathbb{D}}\left|f_{j}^{(n)}(\varphi(z))\right| \\
& =\|u\|_{H_{\mu}^{\infty}} \lim _{j \rightarrow \infty} \sup _{|w| \leq \delta}\left|f_{j}^{(n)}(w)\right|=0 .
\end{aligned}
$$

From this and by Lemma 3.5 we see that the operator $D_{\varphi, u}^{n}: \mathcal{B}^{\alpha} \rightarrow H_{\mu}^{\infty}$ is compact. This also shows that

$$
\left\|D_{\varphi, u}^{n}\right\|_{e, \mathcal{B}^{\alpha} \rightarrow H_{\mu}^{\infty}}=0
$$

From (16), (18), and (19), we get the desired result in the case $\sup _{z \in \mathbb{D}}|\varphi(z)|<1$.

Next, we assume that $\sup _{z \in \mathbb{D}}|\varphi(z)|=1$. Let $\left(L_{j}\right)_{j \in \mathbb{N}}$ be the sequence of operators given in Lemmas 3.2-3.4. Since $L_{j}^{* *}$ is compact on $\mathcal{B}^{\alpha}$, for every $j \in \mathbb{N}$, and $D_{\varphi, u}^{n}$ is bounded from $\mathcal{B}^{\alpha}$ to $H_{\mu}^{\infty}$, then $D_{\varphi, u}^{n} L_{j}^{* *}$ is also compact from $\mathcal{B}^{\alpha}$ to $H_{\mu}^{\infty}$. Hence

$$
\begin{aligned}
& \left\|D_{\varphi, u}^{n}\right\|_{e, \mathcal{B}^{\alpha} \rightarrow H_{\mu}^{\infty}} \leq \limsup _{j \rightarrow \infty}\left\|D_{\varphi, u}^{n}-D_{\varphi, u}^{n} L_{j}^{* *}\right\|_{\mathcal{B}^{\alpha} \rightarrow H_{\mu}^{\infty}} \\
& =\limsup _{j \rightarrow \infty}\left\|D_{\varphi, u}^{n}\left(I-L_{j}^{* *}\right)\right\|_{\mathcal{B}^{\alpha} \rightarrow H_{\mu}^{\infty}} \\
& =\limsup _{j \rightarrow \infty} \sup _{\|f\|_{\mathcal{B}^{\alpha} \leq 1}}\left\|D_{\varphi, u}^{n}\left(I-L_{j}^{* *}\right) f\right\|_{H_{\mu}^{\infty}} \\
& =\limsup _{j \rightarrow \infty} \sup _{\|f\|_{\mathcal{B}^{\alpha}} \leq 1} \sup _{z \in \mathbb{D}} \mu(z)\left|u(z)\left(\left(I-L_{j}^{* *}\right) f\right)^{(n)}(\varphi(z))\right| .
\end{aligned}
$$

For each positive integer $i \geq n$, we define $\mathbb{D}_{i}=\left\{z \in \mathbb{D}: r_{i} \leq|\varphi(z)|<r_{i+1}\right\}$, where $r_{i}$ is given in Lemma 3.1. Let $k$ be the smallest positive integer such that $\mathbb{D}_{k} \neq \emptyset$. Since $\sup _{z \in \mathbb{D}}|\varphi(z)|=1$, $\mathbb{D}_{i}$ is not empty for every integer $i \geq k$ and $\mathbb{D}=\bigcup_{i=k}^{\infty} \mathbb{D}_{i}$, we have

$$
\sup _{\|f\|_{\mathcal{B}^{\alpha}} \leq 1} \sup _{z \in \mathbb{D}} \mu(z)\left|u(z)\left(\left(I-L_{j}^{* *}\right) f\right)^{(n)}(\varphi(z))\right|=I_{1}+I_{2},
$$

where

$$
I_{1}=\sup _{\|f\|_{\mathcal{B}^{\alpha} \leq 1}} \sup _{k \leq i \leq N-1} \sup _{z \in \mathbb{D}_{i}} \mu(z)\left|u(z)\left(\left(I-L_{j}^{* *}\right) f\right)^{(n)}(\varphi(z))\right|
$$


and

$$
I_{2}=\sup _{\|f\|_{\mathcal{B}^{\alpha} \leq 1}} \sup _{N \leq i} \sup _{z \in \mathbb{D}_{i}} \mu(z)\left|u(z)\left(\left(I-L_{j}^{* *}\right) f\right)^{(n)}(\varphi(z))\right| .
$$

Here $N$ is a positive integer determined as follows.

By Lemma 3.1, $\lim _{i \rightarrow \infty} \frac{i^{1-\alpha}}{H_{i, \alpha}\left(r_{i+1}\right)}=\frac{e^{n+\alpha}}{(n+\alpha)^{n+\alpha}}$. Hence, for any given $\varepsilon>0$, there exists an $N \in$ $\mathbb{N}$ such that

$$
\frac{i^{1-\alpha}}{H_{i, \alpha}\left(r_{i+1}\right)} \leq \frac{e^{n+\alpha}}{(n+\alpha)^{n+\alpha}}+\varepsilon
$$

when $i \geq N$. For such $N$ it follows that

$$
\begin{aligned}
& I_{2}=\sup _{\|f\|_{\mathcal{B}^{\alpha}} \leq 1} \sup _{N \leq i} \sup _{z \in \mathbb{D}_{i}} \mu(z)\left|u(z)\left(\left(I-L_{j}^{* *}\right) f\right)^{(n)}(\varphi(z))\right|
\end{aligned}
$$

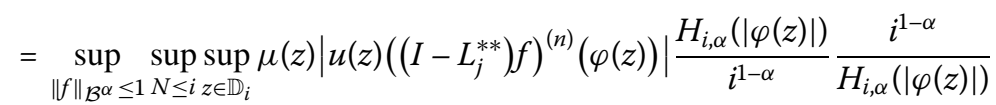

$$
\begin{aligned}
& \preceq\left(\frac{e^{n+\alpha}}{(n+\alpha)^{n+\alpha}}+\varepsilon\right) \sup _{\|f\|_{\mathcal{B}^{\alpha} \leq 1}}\left\|\left(I-L_{j}^{* *}\right) f\right\|_{\mathcal{B}^{\alpha}} \sup _{N \leq i} \sup _{z \in \mathbb{D}_{i}} \mu(z)|u(z)| \frac{i !}{(i-n) !} \frac{|\varphi(z)|^{i-n}}{i^{1-\alpha}} \\
& \preceq\left\|I-L_{j}^{* *}\right\| \sup _{N \leq i} i^{\alpha-1}\left\|D_{\varphi, u}^{n}\left(p_{i}\right)\right\|_{H_{\mu}^{\infty}}
\end{aligned}
$$

Thus

$$
\limsup _{j \rightarrow \infty} I_{2} \preceq \sup _{i \geq N} i^{\alpha-1}\left\|D_{\varphi, u}^{n}\left(p_{i}\right)\right\|_{H_{\mu}^{\infty}}
$$

By Lemmas 3.2, 3.3, 3.4, and Cauchy's integral formula, we have

$$
\begin{aligned}
& \limsup _{j \rightarrow \infty} I_{1}=\limsup _{j \rightarrow \infty} \sup _{\|f\|_{\mathcal{B}^{\alpha} \leq 1}} \sup _{k \leq i \leq N-1} \sup _{z \in \mathbb{D}_{i}} \mu(z)\left|u(z)\left(\left(I-L_{j}^{* *}\right) f\right)^{(n)}(\varphi(z))\right|
\end{aligned}
$$

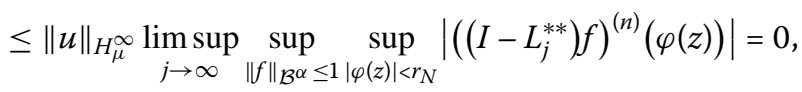

which together with (20) implies that

$$
\begin{aligned}
& \underset{j \rightarrow \infty}{\limsup } \sup _{\|f\|_{\mathcal{B}^{\alpha} \leq 1}} \sup _{z \in \mathbb{D}} \mu(z)\left|u(z)\left(\left(I-L_{j}^{* *}\right) f\right)^{(n)}(\varphi(z))\right| \\
& \preceq \sup _{i \geq N} i^{\alpha-1}\left\|D_{\varphi, u}^{n}\left(p_{i}\right)\right\|_{H_{\mu}^{\infty}} .
\end{aligned}
$$

Therefore

$$
\left\|D_{\varphi, u}^{n}\right\|_{e, \mathcal{B}^{\alpha} \rightarrow H_{\mu}^{\infty}} \preceq \sup _{i \geq N} i^{\alpha-1}\left\|D_{\varphi, u}^{n}\left(p_{i}\right)\right\|_{H_{\mu}^{\infty}}
$$

From the last relation we get

$$
\left\|D_{\varphi, u}^{n}\right\|_{e, \mathcal{B}^{\alpha} \rightarrow H_{\mu}^{\infty}} \preceq \limsup _{i \rightarrow \infty} i^{\alpha-1}\left\|D_{\varphi, u}^{n}\left(p_{i}\right)\right\|_{H_{\mu}^{\infty}} .
$$


From (16), (18), and (21), the asymptotic relations in (15) follow, completing the proof of the theorem.

From Theorem 3.6, letting $\alpha=1$ we deduce the following result.

Corollary 3.7 Let $n$ be a positive integer, $\mu$ a weight, $u \in H(\mathbb{D})$, and $\varphi$ be an analytic selfmap of $\mathbb{D}$. Suppose that $D_{\varphi, u}^{n}: \mathcal{B} \rightarrow H_{\mu}^{\infty}$ is bounded. Then

$$
\left\|D_{\varphi, u}^{n}\right\|_{e, \mathcal{B} \rightarrow H_{\mu}^{\infty}} \approx\left\|D_{\varphi, u}^{n}\right\|_{e, \mathcal{B}_{0} \rightarrow H_{\mu}^{\infty}} \approx \limsup _{j \rightarrow \infty}\left\|D_{\varphi, u}^{n}\left(p_{j}\right)\right\|_{H_{\mu}^{\infty}}
$$

Theorem 3.8 Let $n$ be a positive integer, $\alpha>0, \mu$ a weight, $u \in H(\mathbb{D})$ and $\varphi$ be an analytic self-map of $\mathbb{D}$. If $D_{\varphi, u}^{n}: \mathcal{B}^{\alpha} \rightarrow H_{\mu}^{\infty}$ is bounded, then the following statements are equivalent.

(a) The operator $D_{\varphi, u}^{n}: \mathcal{B}^{\alpha} \rightarrow H_{\mu}^{\infty}$ is compact.

(b) The operator $D_{\varphi, u}^{n}: \mathcal{B}_{0}^{\alpha} \rightarrow H_{\mu}^{\infty}$ is compact.

(c) $\lim _{j \rightarrow \infty} j^{\alpha-1}\left\|D_{\varphi, u}^{n}\left(p_{j}\right)\right\|_{H_{\mu}^{\infty}}=0$.

(d) $\lim _{|\varphi(w)| \rightarrow 1}\left\|D_{\varphi, u}^{n} f_{\varphi(w)}\right\|_{H_{\mu}^{\infty}}=0$.

(e) $\lim _{|\varphi(z)| \rightarrow 1} \frac{\mu(z)|u(z)|}{\left(1-|\varphi(z)|^{2}\right)^{n+\alpha-1}}=0$.

Proof The equivalence of statements (a)-(c) follows from Theorem 3.6.

(c) $\Rightarrow$ (d) From (c), we see that, for every $\varepsilon>0$, there is an $N \in \mathbb{N}$ such that

$$
j^{\alpha-1}\left\|D_{\varphi, u}^{n}\left(p_{j}\right)\right\|_{H_{\mu}^{\infty}}<\varepsilon / 2,
$$

for all $j \geq N$.

Let $\left(z_{k}\right)_{k \in \mathbb{N}} \subset \mathbb{D}$ be an arbitrary sequence such that $\left|\varphi\left(z_{k}\right)\right| \rightarrow 1$ as $k \rightarrow \infty$ (if such a sequence does not exist then the equality in (d) vacuously holds). Similarly to the proof of Theorem 2.1, we have

$$
\begin{aligned}
\left\|D_{\varphi, u}^{n} f_{\varphi\left(z_{k}\right)}\right\|_{H_{\mu}^{\infty}} \leq & C\left(1-\left|\varphi\left(z_{k}\right)\right|^{2}\right) \sum_{j=n}^{\infty}\left|\varphi\left(z_{k}\right)\right|^{j} j^{\alpha-1}\left\|D_{\varphi, u}^{n}\left(p_{j}\right)\right\|_{H_{\mu}^{\infty}} \\
= & C\left(1-\left|\varphi\left(z_{k}\right)\right|^{2}\right) \sum_{j=n}^{N-1}\left|\varphi\left(z_{k}\right)\right|^{j} j^{\alpha-1}\left\|D_{\varphi, u}^{n}\left(p_{j}\right)\right\|_{H_{\mu}^{\infty}} \\
& +C\left(1-\left|\varphi\left(z_{k}\right)\right|^{2}\right) \sum_{j=N}^{\infty}\left|\varphi\left(z_{k}\right)\right|^{j} j^{\alpha-1}\left\|D_{\varphi, u}^{n}\left(p_{j}\right)\right\|_{H_{\mu}^{\infty}} \\
\leq & 2 C\left(1-\left|\varphi\left(z_{k}\right)\right|^{N}\right) M_{0}+C \varepsilon,
\end{aligned}
$$

for $k \in \mathbb{N}$, where $M_{0}=\max _{n \leq j \leq N-1} j^{\alpha-1}\left\|D_{\varphi, u}^{n}\left(p_{j}\right)\right\|_{H_{\mu}^{\infty}}$.

Since $\left|\varphi\left(z_{k}\right)\right| \rightarrow 1$ as $k \rightarrow \infty$, from (22), we deduce that

$$
\limsup _{k \rightarrow \infty}\left\|D_{\varphi, u}^{n} f_{\varphi\left(z_{k}\right)}\right\|_{H_{\mu}^{\infty}} \leq C \varepsilon
$$

Since $\varepsilon$ is an arbitrary positive number, the implication follows.

(d) $\Rightarrow$ (e) Let $\left(z_{k}\right)_{k \in \mathbb{N}}$ be a sequence in $\mathbb{D}$ such that $\lim _{k \rightarrow \infty}\left|\varphi\left(z_{k}\right)\right|=1$ (if such a sequence does not exist then the implication vacuously holds). Since the sequence $\left(f_{\varphi\left(z_{k}\right)}\right)_{k \in \mathbb{N}}$ 
is bounded in $\mathcal{B}^{\alpha}$ and converges to 0 uniformly on compact subsets of $\mathbb{D}$, by (9) and Lemma 3.5, we have

$$
\frac{\mu\left(z_{k}\right)\left|u\left(z_{k}\right) \| \varphi\left(z_{k}\right)\right|^{n} \prod_{j=0}^{n-1}(\alpha+j)}{\left(1-\left|\varphi\left(z_{k}\right)\right|^{2}\right)^{n+\alpha-1}} \leq\left\|D_{\varphi, u}^{n} f_{\varphi\left(z_{k}\right)}\right\|_{H_{\mu}^{\infty}} \rightarrow 0 \quad \text { as } k \rightarrow \infty .
$$

Therefore

$$
\lim _{k \rightarrow \infty} \frac{\mu\left(z_{k}\right)\left|u\left(z_{k}\right)\right|}{\left(1-\left|\varphi\left(z_{k}\right)\right|^{2}\right)^{n+\alpha-1}}=\lim _{k \rightarrow \infty} \frac{\mu\left(z_{k}\right)\left|u\left(z_{k}\right)\right|\left|\varphi\left(z_{k}\right)\right|^{n}}{\left(1-\left|\varphi\left(z_{k}\right)\right|^{2}\right)^{n+\alpha-1}}=0,
$$

which implies (e).

(e) $\Rightarrow$ (a) Assume $\left(f_{k}\right)_{k \in \mathbb{N}}$ is a bounded sequence in $\mathcal{B}^{\alpha}$ converging to 0 uniformly on compact subsets of $\mathbb{D}$. By the assumption, for any $\varepsilon>0$, there exists a $\delta \in(0,1)$ such that

$$
\frac{\mu(z)|u(z)|}{\left(1-|\varphi(z)|^{2}\right)^{n+\alpha-1}}<\varepsilon
$$

when $\delta<|\varphi(z)|<1$.

Therefore, since $u \in H_{\mu}^{\infty}$ we have

$$
\begin{aligned}
\left\|D_{\varphi, u}^{n} f_{k}\right\|_{H_{\mu}^{\infty}}= & \sup _{z \in \mathbb{D}} \mu(z)\left|\left(D_{\varphi, u}^{n} f_{k}\right)(z)\right| \\
\leq & \sup _{z \in \Omega_{\delta}} \mu(z)|u(z)|\left|f_{k}^{(n)}(\varphi(z))\right| \\
& +C \sup _{z \in \mathbb{D} \backslash \Omega_{\delta}} \frac{\mu(z)|u(z)|}{\left(1-|\varphi(z)|^{2}\right)^{n+\alpha-1}}\left\|f_{k}\right\|_{\mathcal{B}^{\alpha}} \\
\leq & \|u\|_{H_{\mu}^{\infty}} \sup _{z \in \Omega_{\delta}}\left|f_{k}^{(n)}(\varphi(z))\right|+C \varepsilon\left\|f_{k}\right\|_{\mathcal{B}^{\alpha}},
\end{aligned}
$$

where $\Omega_{\delta}=\{z \in \mathbb{D}:|\varphi(z)| \leq \delta\}$.

Since $\left(f_{k}\right)_{k \in \mathbb{N}}$ converges to 0 uniformly on compact subsets of $\mathbb{D}$, by Cauchy's estimate so do the sequences $\left(f_{k}^{(n)}\right)_{k \in \mathbb{N}}$ for every $n \in \mathbb{N}$. Letting $k \rightarrow \infty$ in (25) and using the fact that $\varepsilon$ is an arbitrary positive number, we obtain $\lim _{k \rightarrow \infty}\left\|D_{\varphi, u}^{n} f_{k}\right\|_{H_{\mu}^{\infty}}=0$. By Lemma 3.5, we deduce that $D_{\varphi, u}^{n}: \mathcal{B}^{\alpha} \rightarrow H_{\mu}^{\infty}$ is compact.

\section{Competing interests}

The authors declare that they do not have competing interests.

\section{Authors' contributions}

Both authors contributed equally to the writing of this paper. They read and approved the final version of the manuscript.

\section{Author details}

${ }^{1}$ Institute of Systems Engineering, Macau University of Science and Technology, Avenida Wai Long, Taipa, Macau.

${ }^{2}$ Mathematical Institute of the Serbian Academy of Sciences, Knez Mihailova 36/III, Beograd, 11000 , Serbia. ${ }^{3}$ Operator

Theory and Applications Research Group, Department of Mathematics, King Abdulaziz University, P.O. Box 80203, Jeddah, 21589, Saudi Arabia.

\section{Acknowledgements}

Songxiao Li was supported by the Macao Science and Technology Development Fund (No. 083/2014/A2). Stevo Stević was supported by the Serbian Ministry of Science projects III 41025 and III 44006. 


\section{References}

1. Zhu, K: Bloch type spaces of analytic functions. Rocky Mt. J. Math. 23, 1143-1177 (1993)

2. Bierstedt, KD, Summers, WH: Biduals of weighted Banach spaces of analytic functions. J. Aust. Math. Soc. A 54, 70-79 (1993)

3. Stević, S: Norm and essential norm of composition followed by differentiation from $\alpha$-Bloch spaces to $H_{\mu}^{\infty}$. Appl. Math. Comput. 207, 225-229 (2009)

4. Hedenmalm, H, Korenblum, B, Zhu, K: Theory of Bergman Spaces. Graduate Texts in Mathematics, vol. 199. Springer, New York (2000)

5. Zhu, X: Generalized weighted composition operators on weighted Bergman spaces. Numer. Funct. Anal. Optim. 30, 881-893 (2009)

6. Stević, S: Weighted differentiation composition operators from mixed-norm spaces to weighted-type spaces. Appl. Math. Comput. 211, 222-233 (2009)

7. Hibschweiler, R, Portnoy, N: Composition followed by differentiation between Bergman and Hardy spaces. Rocky Mt. J. Math. 35, 843-855 (2005)

8. Li, S, Stević, S: Composition followed by differentiation between Bloch type spaces. J. Comput. Anal. Appl. 9, 195-205 (2007)

9. Li, S, Stević, S: Composition followed by differentiation from mixed-norm spaces to $\alpha$-Bloch spaces. Sb. Math. 199(12), 1847-1857 (2008)

10. Li, S, Stević, S: Composition followed by differentiation between $H^{\infty}$ and $\alpha$-Bloch spaces. Houst. J. Math. 35, 327-340 (2009)

11. Li, S, Stević, S: Products of composition and differentiation operators from Zygmund spaces to Bloch spaces and Bers spaces. Appl. Math. Comput. 217, 3144-3154 (2010)

12. Ohno, S: Products of composition and differentiation between Hardy spaces. Bull. Aust. Math. Soc. 73(2), 235-243 (2006)

13. Stević, S: Products of composition and differentiation operators on the weighted Bergman space. Bull. Belg. Math Soc. Simon Stevin 16, 623-635 (2009)

14. Stević, S: Characterizations of composition followed by differentiation between Bloch-type spaces. Appl. Math. Comput. 218, 4312-4316 (2011)

15. Wu, Y, Wulan, H: Products of differentiation and composition operators on the Bloch space. Collect. Math. 63, 93-107 (2012)

16. Liang, Y, Zhou, Z: Essential norm of the product of differentiation and composition operators between Bloch-type space. Arch. Math. 100, 347-360 (2013)

17. Stević, S: Weighted differentiation composition operators from mixed-norm spaces to the $n$th weighted-type space on the unit disk. Abstr. Appl. Anal. 2010, Article ID 246287 (2010)

18. Stević, S: Weighted differentiation composition operators from $H^{\infty}$ and Bloch spaces to $n$th weighted-type spaces on the unit disk. Appl. Math. Comput. 216, 3634-3641 (2010)

19. Stević, S, Sharma, AK, Bhat, A: Essential norm of products of multiplication composition and differentiation operators on weighted Bergman spaces. Appl. Math. Comput. 218, 2386-2397 (2011)

20. Yang, W, Zhu, X: Generalized weighted composition operators from area Nevanlinna spaces to Bloch-type spaces. Taiwan. J. Math. 16, 869-883 (2012)

21. Zhu, X: Products of differentiation, composition and multiplication from Bergman type spaces to Bers type space. Integral Transforms Spec. Funct. 18, 223-231 (2007)

22. Zhu, X: Generalized weighted composition operators from Bloch spaces into Bers-type spaces. Filomat 26, 1163-1169 (2012)

23. Cowen, CC, MacCluer, BD: Composition Operators on Spaces of Analytic Functions. CRC Press, Boca Raton (1995)

24. Galindo, P, Lindström, M, Stević, S: Essential norm of operators into weighted-type spaces on the unit ball. Abstr. Appl. Anal. 2011, Article ID 939873 (2011)

25. MacCluer, B, Zhao, R: Essential norm of weighted composition operators between Bloch-type spaces. Rocky Mt. J. Math. 33, 1437-1458 (2003)

26. Madigan, K, Matheson, A: Compact composition operators on the Bloch space. Trans. Am. Math. Soc. 347, 2679-2687 (1995)

27. Stević, S: Essential norms of weighted composition operators from the $\alpha$-Bloch space to a weighted-type space on the unit ball. Abstr. Appl. Anal. 2008, Article ID 279691 (2008)

28. Wulan, H, Zheng, D, Zhu, K: Compact composition operators on BMOA and the Bloch space. Proc. Am. Math. Soc 137, 3861-3868 (2009)

29. Zhao, R: Essential norms of composition operators between Bloch type spaces. Proc. Am. Math. Soc. 138, 2537-2546 (2010)

30. Zhu, K: Spaces of Holomorphic Functions in the Unit Ball. Graduate Texts in Mathematics, vol. 226. Springer, New York (2005)

31. Kirillov, AA, Gvishiani, AD: Theorems and Problems in Functional Analysis. Springer, New York (1982) 